\title{
A SHORT PROOF OF THE EXISTENCE OF VECTOR EUCLIDEAN ALGORITHMS
}

\author{
HELAMAN FERGUSON
}

\begin{abstract}
The classical Euclidean algorithm for pairs of real numbers is generalized to real $n$-vectors by $\operatorname{Alg}(n, \mathbf{Z})$. An iteration of $\operatorname{Alg}(n, \mathbf{Z})$ is defined by three steps. Given $n$ real numbers $\operatorname{Alg}(n, \mathbf{Z})$ constructs either $n$ coefficients of a nontrivial integral linear combination which is zero or $n$ independent sets of simultaneous approximations. Either the coefficients will be a column of a $\mathrm{GL}(n, \mathbf{Z})$ matrix or the simultaneous approximations will be rows of $\mathrm{GL}(n, \mathbf{Z})$ matrices constructed by $\operatorname{Alg}(n, \mathbf{Z})$. This algorithm characterizes linear independence of reals over rationals by $\operatorname{GL}(n, \mathbf{Z})$ orbits of rank $n-1$ matrices.
\end{abstract}

Let $x \in \mathbf{R}^{n}$ be a row vector, $n \geq 1, M(n, \mathbf{R})$ the $n \times n$ real matrices, and $I_{n}$ the $n \times n$ identity matrix. An integral vector $b \in \mathbf{Z}^{n}$ is a nearest integral vector to $x \in \mathbf{R}^{n}$ if the corresponding coordinate entries of $b$ and $x$ differ by no more than one half. Let $\hat{A}$ denote the transpose of the matrix $A$. Define the matrix norm of $A$ by $|A|$ where $|A|^{2}=$ Trace $A \hat{A}$, and similarly define $|x|$. This norm is submultiplicative as well as subadditive. Define $m \in \mathbf{Z}^{n}$ to be a relation for $x$ if $m \neq 0$ and $x \hat{m}=0$. The coordinates of $x$ are said to be $\mathbf{Z}$-linearly independent if $x$ has no relation. If $x \neq 0$, set $P=x \hat{x} I_{n}-\hat{x} x$, then $x P=0$ and rank $P=n-1$. Let $\mathrm{GL}(n, \mathbf{Z})$ be the integral $n \times n$ matrices with det $= \pm 1$. Any row or column of any $\operatorname{GL}(n, \mathbf{Z})$ matrix consists of relatively prime integers. $\mathrm{GL}(n, \mathbf{Z})$ acts on $M(n, \mathbf{R})$ by multiplication on the left.

The algorithm $\operatorname{Alg}(n, \mathbf{Z})$ will be defined by a single iteration which replaces a vector, matrix pair $x, P$ by a pair $x A^{-1}, A P$ for the integral matrix $A \in \mathrm{GL}(n, \mathbf{Z})$ as constructed in Steps $1_{n}, 2_{n}$ and $3_{n}$ below. The following notation for $x$ and $P$ will be assumed in this inductive definition of $\operatorname{Alg}(n, \mathbf{Z})$, cf. Step $2_{n}$. Suppose $x \neq 0, x P=0$, rank $P=n-1$ for a real $n \times n$ matrix $P$. If the last entry of $x$ is $t \in \mathbf{R}$ and $t \neq 0$, set $x=(u t, t), u \in \mathbf{R}^{n-1}$. Set $P=\left[\begin{array}{c}W \\ v\end{array}\right]$ where $v$ is the last row of $P$. Note that $x P=0$ implies $u W=-v$.

$\operatorname{Alg}(1, \mathbf{Z}), n=1$. If $x=0$, terminate; otherwise set $A=1$ and replace $x, P$ by $x, P$ where $P=0$.

$\operatorname{Alg}(n, \mathbf{Z}), n>1$. If some entry of $x$ is zero, terminate; otherwise perform the following three steps.

Step $1_{n}$. Let the permutation matrix $E$ exchange a smallest row of $P$ with the last row of $P$. Replace $x, P$ by $x E^{-1}, E P$.

Received by the editors March 5, 1985 and, in revised form, May 31, 1985.

1980 Mathematics Subject Classification. Primary 10E45, 10F10; Secondary 20H05, 10F20, $10 \mathrm{M} 20$.

Key words and phrases. Euclidean algorithm, relations, simultaneous approximations, linear dependence, independence, $\operatorname{GL}(n, \mathbf{Z})$, orbits. 
Step $2_{n}$. Let $Q=u \hat{u} I_{n-1}-\hat{u} u$. Upon $u, Q$ perform $\operatorname{Alg}(n-1, \mathbf{Z})$ until it terminates or $B \in \mathrm{GL}(n-1, \mathbf{Z})$ is constructed such that $|B Q W|<$ $u \hat{u}|v| / 2 \sqrt{n+1}$.

Step $3_{n}$. Let $c$ be a nearest integral vector to $B \hat{u} / u \hat{u}, \hat{c} \in \mathbf{Z}^{n-1}$. Set

$$
A=\left[\begin{array}{cc}
B & c \\
0 & 1
\end{array}\right] \in \mathrm{GL}(n, \mathbf{Z}) .
$$

Replace $x, P$ by $x A^{-1}, A P$.

Case $n=2, \operatorname{Alg}(2, \mathbf{Z})$, is equivalent to the classical Euclidean algorithm. Cf. $[\mathbf{1}, \mathbf{2}]$ for generalized Euclidean algorithms and proofs for all $n \geq 2$ (more complex than the present $\operatorname{Alg}(n, \mathbf{Z}))$. Note that if $\operatorname{Alg}(n, \mathbf{Z})$ terminates, then a relation for $x$ is a column of a $\operatorname{GL}(n, \mathbf{Z})$ matrix constructed by a previous iteration of $\operatorname{Alg}(n, \mathbf{Z})$.

THEOREM. Either $\operatorname{Alg}(n, \mathbf{Z})$ will construct a relation $x \in \mathbf{R}^{n}$ after finitely many iterations or there is no relation for $x$.

Proof. The theorem is true for $n=1$; in this case $\operatorname{Alg}(n, \mathbf{Z})$ simply distinguishes between $x=0$ and $x \neq 0$. Suppose $x \neq 0, x \in \mathbf{R}^{n}$, and consider the pair $x, P$ where $P=x \hat{x} I_{n}-\hat{x} x$. Then $P \hat{m}=(x \hat{x}) m$ if $m$ is any relation for $x$. Since $1 \leq|A \hat{m}|$ for any $A \in \mathrm{GL}(n, \mathbf{Z})$,

$$
0<x \hat{x} \leq|A P||m| .
$$

Assume $n>1$ and that the theorem is true for $\operatorname{Alg}(n-1, \mathbf{Z}), \operatorname{Alg}(n-2, \mathbf{Z}), \ldots$, $\operatorname{Alg}(1, \mathbf{Z})$. Perform one iteration of $\operatorname{Alg}(n, \mathbf{Z})$ upon $x, P$ to construct the matrix $A \in \mathrm{GL}(n, \mathbf{Z})$. Then $x, P$ is replaced by $x A^{-1}, A P$. The matrix of the first $n-1$ rows of the product $A P$ is $B W+c v$. From the definition of $Q$ in Step $2_{n}, W=$ $\hat{u} u W / u \hat{u}+Q W / u \hat{u}$. Hence by this expansion of $W$ in terms of $Q$ and $u W=-v$,

$$
B W+c v=B \hat{u} u W / u \hat{u}+B Q W / u \hat{u}+c v=(c-B \hat{u} / u \hat{u}) v+B Q W / u \hat{u} .
$$

Therefore by the inequality of Step $2_{n}$,

$$
\begin{aligned}
|B W+c v| & \leq|c-B \hat{u} / u \hat{u}||v|+|B Q W / u \hat{u}| \\
& \leq(\sqrt{n-1}+1 / \sqrt{n+1})|v| / 2 .
\end{aligned}
$$

It is supposed that $x, P$ are as after Step $1_{n}$, so that $n|v|^{2} \leq|P|^{2}$. Then

$$
|A P|^{2}=|B W+c v|^{2}+|v|^{2}<\left(n^{2}+6 n+4\right)|P|^{2} / 4 n(n+1) .
$$

Therefore

$$
|A P|<\frac{1}{2} \sqrt{1+(5 / n)}|P| .
$$

Set $M_{0}=I_{n}$ and iterate $\operatorname{Alg}(n, \mathbf{Z}) k$ times upon $x, P$ (initially $P=x \hat{x} I_{n}-\hat{x} x$ ). Let $M_{k} \in \mathrm{GL}(n, \mathbf{Z})$ be the product of the $A$ and $E$ matrices from Steps $3_{n}$ and $1_{n}$ up to and including the $k$ th iteration, $k \geq 0$. If $\operatorname{Alg}(n, \mathbf{Z})$ terminates at the $(k+1)$ st iteration, some entry of $x M_{k}^{-1}$ is zero and a column of $M_{k}^{-1}$ is a relation for $x$. Such a relation will consist of relatively prime integers. Similarly if any of $\operatorname{Alg}(n-1, \mathbf{Z})$, $\operatorname{Alg}(n-2, \mathbf{Z}), \ldots$ terminate, then a relation for $x$ has been constructed. If $\operatorname{Alg}(n, \mathbf{Z})$ never terminates for $x$, then from the second inequality $(* *) M_{k} P$ tends to zero as $k$ increases without bound. Since the first inequality $(*)$ is true for any relation $m \in \mathbf{Z}^{n},|m|$ cannot remain bounded and there are no relations for $x$. 
COROLLARY 1. If $x \in \mathbf{R}^{n}$ has no relation, then for every $\varepsilon>0 \operatorname{Alg}(n, \mathbf{Z})$ constructs $A \in \mathrm{GL}(n, \mathbf{Z})$ with each row less than distance $\varepsilon$ from the line $\mathbf{R} x$.

ProOF. $(x \hat{x}) A=(A \hat{x}) x+A P$ is an orthogonal decomposition of $A$ where $x \hat{x} I_{n}=\hat{x} x+P$. Set $A=M_{k}$ for the $k$ th iteration of $\operatorname{Alg}(n, \mathbf{Z})$ on $x$. Since $\operatorname{Alg}(n, \mathbf{Z})$ never terminates then by the second inequality $(* *)$ above, $M_{k} P$ tends to zero as $k$ increases. Specifically, if $k>(\log (|P| / \varepsilon)) /(\log (2 / \sqrt{1+(5 / n)})$, then $\left|M_{k} P\right|<\varepsilon$.

COROLLARY 2. The closure of the $\mathrm{GL}(n, \mathbf{Z})$ orbit of a rank $n-1$ matrix $P$ contains the zero matrix if and only if the coordinates of any eigenvector corresponding to zero of $P$ are $\mathbf{Z}$-linearly independent.

PROOF. Let $P$ have rank $n-1$ and let $x, 0 \neq x \in \mathbf{R}^{n}$, be an eigenvector of $P$ so that $x P=0$. The if direction: there is no relation for $x$. Hence the algorithm $\operatorname{Alg}(n, \mathbf{Z})$ applied to $x, P$ never terminates. Then matrices $A \in \mathrm{GL}(n, \mathbf{Z})$ are constructed by iteration of $\operatorname{Alg}(n, \mathbf{Z})$ such that by the second inequality $(* *)$ the norm $|A P|$ and hence $A P$ is arbitrarily small. The only if direction: the zero matrix is in the closure of $\mathrm{GL}(n, \mathbf{Z}) P$; i.e., there are $A \in \mathrm{GL}(n, \mathbf{Z})$ such that $A P$ is arbitrarily small. By the first inequality $(*)$ this contradicts the existence of a relation for $x$.

\section{REFERENCES}

1. Helaman Ferguson and Rodney Forcade, Generalization of the Euclidean algorithm for real numbers to all dimensions higher than two, Bull. Amer. Math. Soc. (N.S.) 1 (1979), 912-914.

2. __ Multidimensional Euclidean algorithms, J. Reine Angew. Math. 334 (1982), 171181.

Department of Mathematics, Brigham Young University, Provo, Utah 84602 